\title{
Strong Interaction Fairness via Randomization
}

\author{
Yuh-Jzer Joung* \\ Department of Information Management \\ National Taiwan University \\ Taipei, Taiwan \\ joung@ccms.ntu.edu.tw
}

\author{
Scott A. Smolka ${ }^{\dagger}$ \\ Department of Computer Science \\ SUNY at Stony Brook \\ Stony Brook, NY 11794-4400, U.S.A. \\ sas@cs . sunysb . edu
}

\begin{abstract}
We present Multi, a symmetric, fully distributed, randomized algorithm that, with probability 1, schedules multiparty interactions in a strongly fair manner. To our knowledge, Multi is the first algorithm for sitrong interaction fairness to appear in the literature. Moreover, the expected time taken by Multi to establish an interaction is a constant not depending on the total number of processes in the system. In this sense, Multi guarantees real-time response.

Multi makes no assumptions (other than boundedness) about the time it takes processes to communicate. It thus offers an appealing tonic to the impossibility results of Thay\&B Bagrodia and Joung concerning strong interaction fairness in an environment, shared-memory or message-passing, in which processes are deterministic and the communication time is nonnegligible.

Because strong interaction fairness is as strong a fairness condition that one might actually want to impose in practice, our results indicate that randornization may also prove fruitful for other notions of fairness lacking deterministic realizations and requiring realtime response.
\end{abstract}

\section{Introduction}

A multiparty interaction is a set of $\mathrm{I} / \mathrm{O}$ actions executed jointly by a number of processes, each of which must be ready to execute its own action for any of the actions in the set to occur. An attempt to participate in an interaction delays a process until all other participants are available. After the actions are executed, the participating processes continue their local computation. Although a relatively new concept, the mul-

${ }^{*}$ Researcin supported by the National Science Council, Taipei, Taiwan, under Grant NSC 84-2213-E-002-005.

${ }^{\dagger}$ Research supported in part by NSF Grants CCR-9120995 and CCR-9208585, and AFOSR Grant F49620-93-1-0250. tiparty interaction has found its way into various distributed programming languages and algebraic models of concurrency. See [10] for a taxonomy of programming languages offering linguistic support for multiparty interaction.

Although multiparty interactions are executed synchronously, the underlying model of communication is usually asynchronous and bipartied. The multiparty interaction scheduling problem then is concerned with synchronizing asynchronous processes to satisfy the following requirements: (1) an interaction can be established only if all of its participants are ready (i.e., the interaction is enabled), and (2) a process can participate in only one interaction at a time. Moreover, some notion of fairness is typically associated with the implementation to prevent "unfair" computations that favor a particular process or interaction.

Several important fairness notions have been proposed in the literature $[1,2,3]$, including: weak interaction fairness, where if an interaction is continually enabled, then some of its participants will eventually engage in an interaction; and strong interaction fairness, where an interaction that is infinitely often enabled will be established infinitely often. A distinguishing characteristic of weak interaction fairness is that it is much weaker than most known fairness notions, while strong interaction fairness is much stronger.

In general, stronger fairness notions induce more liveness properties, but are also more difficult to implement. Therefore, it is not surprising to see that only weak interaction fairness has been widely implemented (e.g., $[16,14,4,13,11,17,9]$ ). It is also not surprising to see that all of these algorithms are asymmetric and deterministic, as weak interaction fairness (and thus strong interaction fairness) has been proven impossible by any symmetric, deterministic, and distributed algorithm $[7,12]$. Given that a process decides autonomously when it will attempt an interaction, and at a time that cannot be predicted in advance, strong interaction fairness is still not possible even if the sym- 
metry requirement is dropped $[18,8]$.

Note that these impossibility results do not depend on the type of communication primitives (e.g., message-passing or shared-memory) provided by the underlying execution model. They hold as long as one process's readiness for multiparty interaction can be known by another only through communications, and the time it takes two processes to communicate is nonnegligible (but can be finitely bounded).

In the case of CSP communication guard scheduling, a special case of the multiparty interaction scheduling problem where each interaction involves exactly two processes, randomization has proven to be an effective technique for coping with the aforementioned impossibility phenomena. For example, the randomized algorithm of Reif and Spirakis [15] is symmetric, weak interaction fair with probability 1 , and guarantees real time response: if two processes are continuously willing to interact with each other within a time interval $\Delta$, then they establish an interaction within $\Delta$ time with high likelihood, and the expected time for establishment of interaction is constant.

The randomized algorithm of Francez and Rodeh [7] is simpler: a process $p_{i}$ expresses its willingness to establish an interaction with a process $p_{j}$ by setting a Boolean variable shared by the two processes; $p_{i}$ may then need to wait a certain amount of time $\delta$ before re-accessing the variable to determine if $p_{j}$ is likewise interested in the interaction. The authors show that, under the proviso that the time to access a shared variable is negligible compared to $\delta$, the algorithm is weak interaction fair with probability 1 . Note, however, that this assumption, combined with the fact that no lower bound on $\delta$ is provided, may significantly limit the algorithm's practicality. Furthermore, no strcng interaction fairness is claimed by either algorithm.

In this paper, we present Multi, an extension of Francez and Rodeh's randomized algorithm to the multiparty case. We prove that Multi is weak interaction fair with probability 1 . We also show that if the transition of a process to a state in which it is ready for interaction is independent of the random draws of the other processes, then, with probability 1 , Multi is strong interaction fair. To our knowledge, Multi is the first algorithm for strong interaction fairness to appear in the literature.

We also present a detailed timing analysis of Multi and establish a lower bound on how long a process must wait before re-accessing a shared variable. Consequently, our algorithm can be fine-tuned for optimal performance. Moreover, we show that the expected time to establish an interaction is a constant not depending on the total number of processes in the system. Thus, Multi also guarantees real-time response.
Because strong interaction fairness is as strong a fairness condition that one might actually want to impose in practice, our results indicate that randomization may also prove fruitful for other notions of fairness lacking deterministic realizations and requiring realtime response.

The rest of the paper is organized as follows. Section 2 describes the multiparty interaction scheduling problem in a more anthropomorphic setting, known as Committee Coordination. Our randomized algorithm is presented in Section 3 and analyzed in Section 4. Section 5 concludes.

\section{The Committee Coordination Prob- lem}

The problem of scheduling multiparty interactions in asynchronous systems has been elegantly characterized by Chandy and Misra as one of Committee Coordination [5]:

Professors (cf. processes) in a certain university have organized themselves into committees (cf. interactions) and each committee has a fixed membership roster of one or more professors. From time to time, a professor may decide to attend a committee meeting; it starts waiting and continues to wait until a meeting of a committee of which it is a member is started.

Given that all meetings terminate in finite time, the problem is to devise an algorithm satisfying the following three requirements:

Synchronization: A committee meeting may be started only if all members of that committee are waiting.

Exclusion: No two committees meet simultaneously if they have a common member.

Weak Interaction Fairness: If all professors of a committee are waiting, then eventually some professor will attend a committee meeting.

We shall also consider strong interaction fairness, i.e., a committee that is infinitely often enabled will be started infinitely often. A committee is enabled if every member of the committee is waiting, and is disabled otherwise.

The overall behavior of a professor can be described by the state transition diagram of Figure 1, where state $T$ corresponds to thinking, $W$ corresponds to waiting for a committee meeting, and $E$ means that the professor is actively engaged in a meeting. 


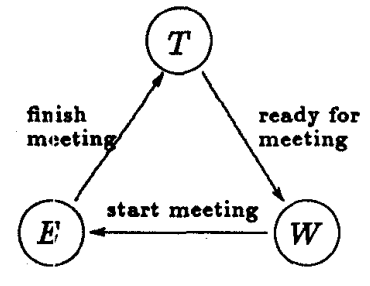

Figure 1: State transition diagram of a professor.

Note that any algorithm for the problem should only control the transition of a professor from state $W$ to state $E$, but not the other two transitions. That is, the transitions from $T$ to $W$ and from $E$ to $T$ are autonomous to each professor. Moreover, we do not assume any upper bound on the time a professor can spend in thinkinis. Otherwise, an algorithm for the problem could simply wait long enough until all professors become waiting, and then schedule a committee meeting of its choosing. All three requirements for the problem and stroing interaction fairness would theln be easily satisfied.

\section{The Algorithm}

In this section, we present Multi, our randomized algorithm for coinmittee coordination. In the algorithm, we associate with each committee $M$ a counter $C_{M}$ whose value ranges over $[0 \cdots \mid$ prof. $M \mid-1]$, where prof. $M$ is the set of professors involved in $M . C_{M}$ can be accessed only by the professors in prof. $M$ and only through the TEST\&OP instruction as follows:

$$
\text { result }:=\operatorname{TEST\& 0P}\left(C_{M}, \text { zero-op, nonzero-op }\right)
$$

The effect of this instruction is to apply to $C_{M}$ the operation zero-op if its value was zero and the operation nonzero-op otherwise, and to assign to the variable result the old value (i.e., the value before the operation) of $C_{M}$. The operations used here are no-op, inc, and dec, where

$$
\begin{aligned}
& \text { no-op }\left(C_{M}\right)=C_{M}, \\
& \operatorname{inc}\left(C_{M}^{\prime}\right)=\left(C_{M}+1\right) \bmod \mid \text { prof. } M \mid \\
& \operatorname{dec}\left(C_{M}^{\prime}\right)=\left(C_{M}-1\right) \bmod \mid \text { prof. } M \mid
\end{aligned}
$$

For example, if $C_{M}=2$, then $\operatorname{TEST} \operatorname{OP}\left(C_{M}\right.$, no-op, dec $)$ sets $C_{M}=1$ and returns 2 . If $C_{M}=0$, $\operatorname{TESTrOP}\left(C_{M}\right.$, no-op, dec) keeps $C_{M}$ unchanged and returns 0 . We assume that the execution of the TESTEDP instruction is "atomic," although, in the full version of the paper, we show how this assumption can be removed.

Algorithm Multi can be informally described as follows. Initially, all the shared counters are set to zero. When a professor: $p_{i}$ decides to attend a committee

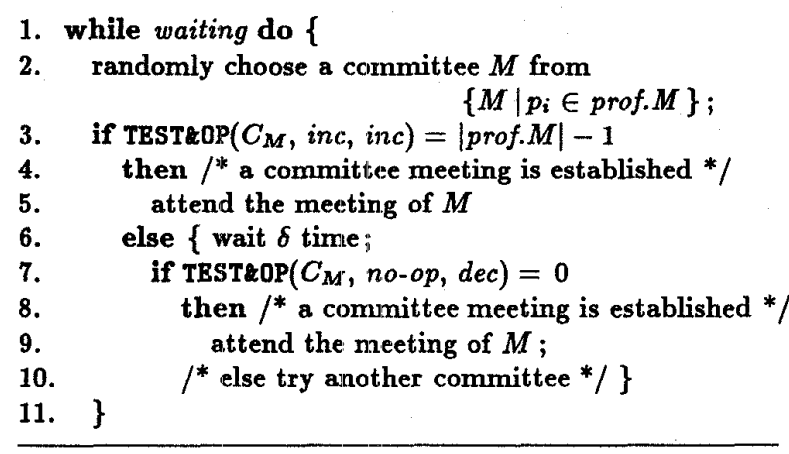

Figure 2: Algorithm Multi for professor $p_{i}$.

meeting, it randomly chooses a committee $M$ of which it is a member. It then attempts to start a meeting of $M$ by increasing the value of $C_{M}$ by 1 (all increments and decrements are to be interpreted modulo (prof. $M \mid$ ). If the new value of $C_{M}$ is 0 (i.e., $C_{M}=\mid$ prof. $M \mid-1$ before the increment), then professor $p_{i}$ concludes that each of the other members of $M$ have increased $C_{M}$ by one, and are waiting for $p_{i}$ to convene $M$. So $p_{i}$ goes to state $E$ to start the meeting.

If the new value of $C_{M}$ is not zero, however, then at least one of the professors in prof. $M$ is not yet ready. So professor $p_{i}$ waits for some period of time (hoping that its partners will become ready) and then re-accesses $C_{M}$. If $C_{M}$ has now been set to 0 , then all the professors which were not ready for $M$ are now ready, and so $p_{i}$ can attend the meeting. If $C_{M}$ is still not zero, then some professor is still not ready for $M$. So $p_{i}$ withdraws its attempt to start $M$ by decreasing the value of $C_{M}$ by 1 and tries another committee.

The algorithm to be executed by each professor $p_{i}$ is presented in Figure 2, where waiting (line 1) is a Boolean flag indicating whether or not $p_{i}$ is waiting for a committee meeting. The constant $\delta$ (line 6 ) is the amount of time a professor waits before re-accessing a counter. We will later require (see Section 4) that $\delta$ be greater than $\eta_{\max } \times(\mid$ prof. $M \mid-1)$, where $\eta_{\max }$ is the maximum amount of time a professor can spend in executing lines 2 and $3 .^{1}$ Note that the algorithm is symmetric in the sense that all professors execute the same code and make no use of their process ids.

\footnotetext{
${ }^{1}$ More precisely, $\eta_{\max }$ should also include the time it takes to execute line 1 . To simplify the analysis, we assume that the Boolean flag waiting only serves to indicate the state of the executing professor, and so no explicit test of the flag is needed. Moreover, we assume that an action is instantaneously executed at some time instance. The time it takes to execute an action is the difference between the tine the action is executed and the time the previous action (of the same professor) was executed.
} 


\section{Analysis of the Algorithm}

In this section we prove that Multi satisfies the synchronization and exclusion requirements of the Committee Coordination problem, and, with probability 1 , is weak and strong interaction fair. We also analyze the expected time Multi takes to schedule a committee meeting.

\subsection{Definitions}

We assume a discrete global time axis where, to an external observer, the events of the system are totally ordered. Internally, however, processors may execute instructions simultaneously at the same time instance. Simultaneous access to a shared counter will be arbitrated in the implementation of the TEST\&OP instruction, which we assume is executed atomically.

Since the time axis is discrete, it is meaningless to say that "there are infinitely many time instances in some finite time interval such that ..." Therefore, throughout this paper, the phrase "there are infinitely many time instances" refers to the interval $[0, \infty)$.

For analysis purposes, we present in Figure 3 a refinement of the state transition diagram of Figure 1 , where state $W$ is refined into three sub-states $W_{0}-W_{2}$. The actions taken by the professors from these substates are:

$W_{0}$ : randomly choose a new committee.

$W_{1}$ : access counter $C_{M}$ by TEST\&OP $\left(C_{M}\right.$, inc, inc).

$W_{2}$ : wait $\delta$ time before re-accessing $C_{M}$ by TEST\&OP( $C_{M}$, no-op, dec).

We say that a professor is watching for committee $M$ if it is in state $W_{2}$ waiting for re-access to $C_{M}$.

According to the algorithm, if at time $t$ a professor $p$ accesses a counter $C_{M}$ by $\operatorname{TEST\& OP}\left(C_{M}\right.$, inc, inc $)$ in state $W_{1}$, then it will be in state $W_{2}$ or $E$ after $t$, depending on the value of $C_{M}$. Furthermore, if $p$ enters state $W_{2}$ at time $t$ to watch for a committee $M$, then at time $t+\delta$ it will re-access $C_{M}$ by TEST\&OP $\left(C_{M}\right.$, no$o p, d e c)$. Depending on the value of $C_{M}$, after time $t+\delta$ the professor will either return to state $W_{0}$ to choose another committee, or enter state $E$ to attend the meeting of $M$.

Figure 4 illustrates a possible scenario for four professors $p_{1}, p_{2}, p_{3}$, and $p_{4}$ executing the algorithm, where $p_{1}$ and $p_{4}$ are involved in committee $M_{14}, p_{1}, p_{2}$, and $p_{3}$ are involved in $M_{123}$, and $p_{2}, p_{3}$ and $p_{4}$ are involved in $M_{234}$. For each professor, we explicitly depict its state (on the $\mathrm{Y}$-axis) at each global time instance (on the $\mathrm{X}$-axis). For example, at time 1 professor $p_{1}$ starts waiting for a committee meeting and so it enters state $W_{0}$ from state $T$. At time 2, it randomly chooses

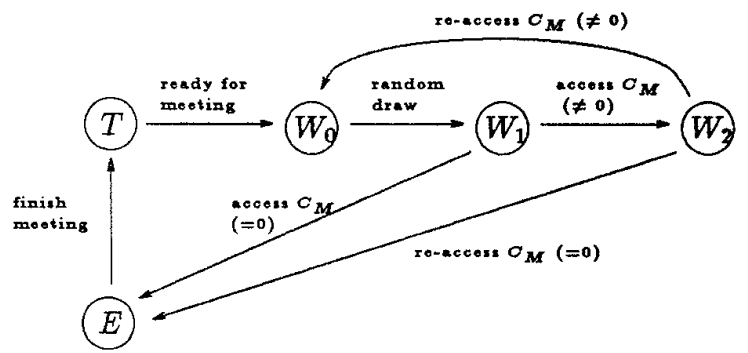

Figure 3: State transition diagram of a professor executing the algorithm.

$M_{14}$ and then accesses $C_{M_{14}}$ at time 3. Since $C_{M_{14}}=0$ before the access, $p_{1}$ enters state $W_{2}$ to watch for $M_{14}$ for $\delta=3$ time units and then re-accesses $C_{M_{14}}$ at time 6. Since $p_{4}$ will not access $C_{M_{14}}$ until time $9, p_{1}$ returns to state $W_{0}$ to try another committee. Later at time $12, p_{1}$ chooses committee $M_{123}$ and then accesses $C_{M_{123}}$ at time 13 . When $p_{1}$ re-accesses $C_{M_{123}}$ at time 16 , it finds that both $p_{2}$ and $p_{3}$ are willing to start the meeting of $M_{123}$. So $p_{1}$ enters state $E$ to attend the meeting. The meeting of $M_{123}$ ends at time 19, after which the committee members can return to state $T$ at a time of their own choosing. The shaded area between time 17 and 19 represents a synchronization interval for the three professors.

\subsection{Properties of the Algorithm That Hold with Certainty}

We now analyze the correctness of the algorithm. We begin with an invariant about the value of a shared counter $C_{M}$, which we will use in proving that Multi satisfies the synchronization condition of the Committee Coordination Problem.

Lemma 1 If at time $t$ there are $k$ professors in state $W_{2}$ watching for committee $M$ and no professor, since last entering state $W_{0}$, has entered state $E$ to attend a meeting of $M$, then the value of $C_{M}$ at time $t$ is $k$. If, however, at time $t$ some professor has entered state $E$ to attend a meeting of $M$, then $C_{M}=0$ and in $[t, t+\delta]$ all professors in prof. $M$ will have entered state $E$ to attend the meeting of $M$.

Proof: By induction on $t_{i}$, the time at which the $i$ th system event occurs.

Theorem 1 (Synchronization) If a professor in prof. $M$ enters state $E$ at time $t$ to attend a meeting of $M$, then within $\delta$ time all professors in prof. $M$ will have entered state $E$ to attend the meeting of $M$. 


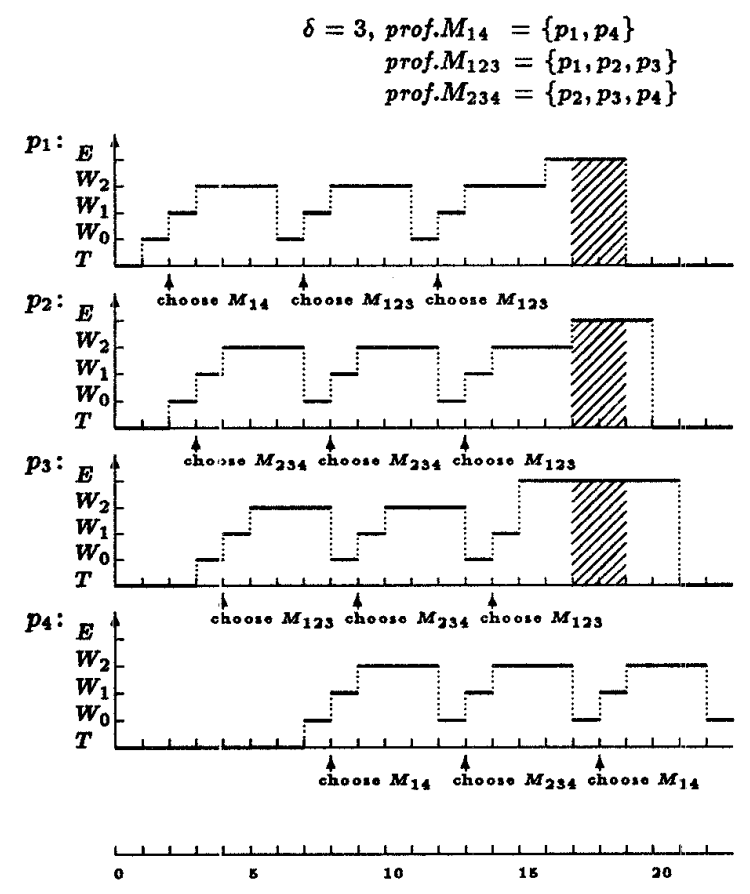

Figure 4: A partial computation of four professors.

Proof: The theorem follows immediately from Lemma 1.

Theorem 2 (Exclusion) No two committees convene simultaneously if they have a common member.

Proof: The result follows from the fact that a professor watches for one committee at a time.

\subsection{Properties of the Algorithm That Hold with Probability 1}

We move on to prove that Multi is weak and strong interaction fair, and analyze its time complexity. For this we will need some definitions about the "random draw" a professor performs in state $W_{0}$ when deciding which meeting to attempt.

We say that a professor accesses a counter $C_{M}$ when it executes the instruction $\operatorname{TEST\& OP}\left(C_{M}\right.$, inc, inc $)$ (line 3 of the algorithm) and re-accesses $C_{M}$ when it executes TEST\&CP $\left(C_{M}\right.$, no-op, dec) (line 7). Now suppose that professor $p$ accesses some counter in the time interval $(t-\delta, t]$. If there is more than one such access, choose the most recent one. Then the choice of counter must be the result of the random draw performed before the access (line 2). Let $D_{t, p}$ denote this random draw, and let $D_{t, p r o f . M}=\left\{D_{t, p} \mid p \in\right.$ prof. $M$ and $D_{t, p}$ is defined $\}$.
Note that if $p$ is in state $W_{2}$ at time $t$, then $p$ must have accessed some counter in the interval $(t-\delta, t]$, and so $D_{t, p}$ must be defined. Note further that the interval $(t-\delta, t]$ is open at $t-\delta$ and closed at $t$. Therefore, $D_{t, p}$ is defined if $p$ accesses $C_{M}$ at $t$, but is not defined if the access occurs at $t-\delta$. The reason for choosing this semi-closed interval is to avoid the situation where an access to a counter occurs simultaneously with a re-access to the same counter by another professor.

For example, suppose that counter $C_{M}$, shared exclusively by $p_{1}$ and $p_{2}$, is accessed by $p_{1}$ at time $t-\delta$ and found to be zero. Then $p_{1}$ must wait $\delta$ time before re-accessing $C_{M}$ at time $t$. Suppose further that $p_{2}$ also chooses $M$ and accesses $C_{M}$ at time $t$. We thus have an access/re-access conflict involving $C_{M}$ at time $t$, and the meeting is established if and only if the conflict is resolved in favor of the access; i.e., $p_{2}$ gets to go first.

Note, however, that no dependency on the resolution order is manifest if $p_{1}$ 's access occurs any time after $t-\delta$ (but no later than $t$ ). In this case, $p_{2}$ 's access is certain to precede $p_{1}$ 's re-access and the meeting is once again established. A.lso noteworthy of this case is the fact that both $D_{t, p_{1}}$ and $D_{t, p_{2}}$ are defined, while in the case where $p_{1}$ 's access occurs at $t-\delta$, only $D_{t, p_{z}}$ is defined. In general, if $D_{t, p}$ is defined for all $p \in M$, and these random draws yield the same outcome $M$, then $M$ will be established (see Lemma 4).

In the rest of this section we shall use $\psi_{p, M}$ to denote the fixed non-zero probability that professor $p \in$ prof. $M$ chooses committee $M$ in a random draw. Thus,

$$
\psi_{M}=\prod_{p \in p r o f . M} \psi_{p, M}
$$

is the probability that a set of mutually independent random draws, one by each professor in prof. $M$, yields the same outcome $M$.

The following three lemmas are used in the fairness proofs.

Lemma $2 \forall t, t^{\prime}$ such that $t^{\prime}-t>\delta$, $D_{t, \text { prof.M }} \cap D_{t^{\prime}, \text { prof.M }}=\emptyset$.

Proof: Follows directly from the definition of $D_{t, p}$.

Lemma 3 Suppose that committee $M$ is continuously enabled in the interval $\left(t_{i}, t_{i}+\theta\right)$. Then there exists a time instance $t, t_{i}<t \leq t_{i}+\theta$, such that $\left|D_{t, \text { prof. } M}\right|=\mid$ prof. $M \mid$ if:
1. $\theta \geq \eta_{\max } \times \mid$ prof. $M \mid$, and
2. $\delta>\eta_{\max } \times(\mid$ prof. $M \mid-1)$. 
Proof: Since $M$ is enabled in $\left(t_{i}, t_{i}+\theta\right)$, every professor of $M$ is in a $W$-state in this interval. Clearly, either (i) there exists an interval $\left(t_{i}, t_{j}\right), t_{i}<t_{j} \leq t_{i}+\theta$, during which every professor $p \in$ prof. $M$ is in state $W_{2}$, or (ii) there exists some professor $p$ such that $p$ is not in state $W_{2}$ in $\left(t_{i}, t_{j}\right)$. In case (i), $D_{t, p}$ must be defined for every $p \in$ prof. $M$ and every $t$ in $\left(t_{i}, t_{j}\right)$, and so $\left|D_{t, p r o f . M}\right|=\mid$ prof. $M \mid$.

For case (ii), suppose that some professor $p_{1} \in$ prof. $M$ stays in $W_{0}$ or $W_{1}$ in $\left(t_{i}, t_{i}+m_{1}\right)$ and then accesses some counter at $t_{i}+m_{1}$, where $m_{1}>0$. So $D_{t_{i}+m_{1}, p_{1}}$ is defined. Since $m_{1} \leq \eta_{\max }$ and $\mid$ prof. $M \mid \geq$ 1 , by condition $1, t_{i}<t_{i}+m_{1} \leq t_{i}+\theta$.

If $D_{t_{i}+m_{1}, p}$ is defined for all $p \in$ prof.M (i.e., $\left|D_{t_{i}+m_{1}, p r o f . M}\right|=\mid$ prof. $\left.M \mid\right)$, then we are done. Otherwise, $\mid$ prof. $M \mid$ must be greater than one, and there exists another professor $p_{2}$ such that $D_{t, p_{2}}$ is not defined for all $t$ in $\left[t_{i}+m_{1}, t_{i}+m_{1}+m_{2}\right)$, but $D_{t, p_{2}}$ is defined for $t=t_{i}+m_{1}+m_{2}$ (similarly because $p_{2}$ stays in state $W_{0}$ or $W_{1}$ in $\left(t_{i}+m_{1}, t_{i}+m_{1}+m_{2}\right)$, and then accesses some counter at $\left.t_{i}+m_{1}+m_{2}\right)$. Moreover, $0<m_{2} \leq \eta_{\max }$.

By condition 1 and the fact that $\mid$ prof. $M \mid \geq 2$, we have $m_{1}+m_{2} \leq \theta$. So $p_{1}$ is still in a $W$-state in $\left(t_{i}+\right.$ $\left.m_{1}, t_{i}+m_{1}+m_{2}\right)$. However, since $p_{1}$ accesses some counter at $t_{i}+m_{1}, p_{1}$ must have entered state $W_{2}$ after the access, and stays in $W_{2}$ in $\left(t_{i}+m_{1}, t_{i}+m_{1}+\delta\right)$. So $D_{t, p_{1}}$ must be defined for all $t$ in $\left[t_{i}+m_{1}, t_{i}+m_{1}+\delta\right)$. Moreover, by condition $2, t_{i}+m_{1}<t_{i}+m_{1}+m_{2}<$ $t_{i}+m_{1}+\delta$. So $D_{t, p}$ must be defined for both $p=$ $p_{1}, p_{2}$, and $t=t_{i}+m_{1}+m_{2}$. Since $m_{1}+m_{2} \leq \theta$, $t_{i}<t_{i}+m_{1}+m_{2} \leq t_{i}+\theta$.

If $D_{t_{i}+m_{1}+m_{2}, p}$ is also defined for every other professor $p \in$ prof. $M$, then we are done. Otherwise, prof. $M$ must contain at least three professors, and there exists a third professor $p_{3}$ such that $D_{t, p_{3}}$ is not defined for all $t$ in $\left[t_{i}+m_{1}+m_{2}, t_{i}+m_{1}+m_{2}+m_{3}\right)$, where $0<m_{3} \leq \eta_{\max }$, but $D_{t, p_{3}}$ is defined for $t=$ $t_{i}+m_{1}+m_{2}+m_{3}$. Moreover, we can use a similar argument to show that $D_{t, p_{1}}$ and $D_{t, p_{2}}$ must also be defined for $t=t_{i}+m_{1}+m_{2}+m_{3}$. In general, we can show that if $\mid$ prof. $M \mid \geq n$, then $D_{t, p_{k}}$ must be defined for $k=1 . . n$ and $t_{i}<t=t_{i}+m_{1}+m_{2}+\ldots+m_{n} \leq t_{i}+\theta$.

Since the number of professors in prof. $M$ is finite, eventually we will reach a point where $D_{t, p}$ is defined for each $p \in$ prof. $M$ and some $t_{i}<t \leq t_{i}+\theta$ (see Figure 5). The lemma is then established.

We shall henceforth refer to Condition 2 of Lemma 3 (the lower bound on $\delta$ ) as Assumption A1. Note that different professors can choose different values for $\delta$; these values need only satisfy the lower bound established by the lemma. As such, the clocks used by the professors to implement time-outs need not be adjusted

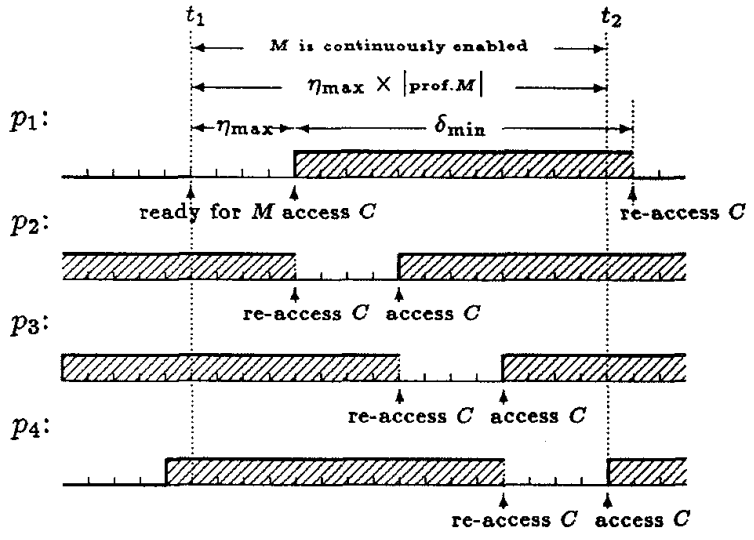

Figure 5: Hllustration of Lemma 3. $\left(t_{1}, t_{2}\right)$ is the maximum possible interval during which $M$ is enabled but $\left|D_{t, \text { prof. } M}\right| \neq \mid$ prof. $M \mid$. Here $\mid$ prof. $M \mid=4$. Then, $D_{t_{2}, p}$ must be defined for all $p \in$ prof. $M$. Note that if $\delta_{\min }$ would equal $\eta_{\max } \times(\mid$ prof. $M \mid-1)$, then $D_{t_{2}, p_{1}}$ would not be defined.

to the same accuracy.

Lemma 3 says that if a committee $M$ is continuously enabled sufficiently long, then there exists an interval of length $\theta$ within which every professor in prof. $M$ performs a random draw. The following lemma ensures that if their random draws yield the same outcome, then they must establish $M$.

Lemma 4 If $\left|D_{t, p r o f . M}\right|=\mid$ prof. $M \mid$ and all the random draws in $D_{t, p r o f . M}$ yield the same outcome $M$, then by time $t$ some professor must have already entered state $E$ to start $M$, and by time $t+\delta$ all professors in prof. $M$ will enter state $E$ to start $M$.

Proof: Assume the hypotheses described in the lemma. Let $p_{i} \in$ prof. $M$ be the first professor which, after performing its random draw in $D_{t, p r o f . M}$, accesses $C_{M}$ by TEST\&OP $\left(C_{M}\right.$, inc, inc $)$, and let $p_{j} \in$ prof. $M$ be the last professor to do so. Let $t_{i}$ and $t_{j}$ be the time at which $p_{i}$ and $p_{j}$, respectively, accesses $C_{M}$. Then, $t_{j}-t_{i}<\delta$, and $t-\delta<t_{i} \leq t_{j} \leq t$.

Since $p_{i}$ accesses $C_{M}$ at time $t_{i}$, it will not re-access $C_{M}$ until $t_{i}+\delta$. Since $t_{i}+\delta>t$, all professors which access $C_{M}$ in $\left[t_{i}, t_{j}\right]$ will remain in state $W_{2}$ before $p_{j}$ accesses $C_{M}$. By Lemma $1, C_{M}=\mid$ prof. $M \mid-1$ just before $p_{j}$ 's access. So when $p_{j}$ accesses $C_{M}$ at $t_{j}$, it will set $C_{M}$ to zero and enter state $E$ to start $M$. Moreover, by time $t_{j}+\delta$, every other professor of $M$ will learn that $M$ has been started when it re-accesses $C_{M}$ by $\operatorname{TEST\& OP}\left(C_{M}, n o-o p, d e c\right)$, and so will also enter state $E$ to start $M$. Since $t_{j}+\delta \leq t+\delta$, the lemma is thus established. 
Theorem 3 (Weak Interaction Fairness)

Assume $A 1$ and that all members of a committee $M$ are waiting for committee meetings. Then, the probability is 1 thai eventually a meeting involving some member of $M$ will be started.

Proof: Suppose that $M$ is enabled at $t$. If $M$ is continuously enabled in $\left(t, t+\eta_{\max } \times \mid\right.$ prof. $\left.M \mid\right)$, then by Lemma 3 under $A 1$, there exists a time instance $t_{1}$, $t<t_{1} \leq t+\eta_{\mathrm{mex}} \times \mid$ prof. $M \mid$, such that $\left|D_{t_{1}, \text { prof. } M}\right|=$ |prof.M|. If the random draws in $D_{t_{1}, \text { prof.M }}$ yield the same outcome $M$, then, by Lemma $4, M$ must be disabled at $t_{1}$. Even if the random draws do not yield the same outcome, some professor of $M$ may still establish another committee meeting $M^{\prime}$ if its random draw has the outcome $M^{\prime}$ and at the same time all other professors of $M^{\prime}$ are also interested in $M^{\prime}$. So the probability that the random draws in $D_{t_{1}, p r o f . M}$ do not cause any committee involving a member of $M$ to be started is no greater than $1-\psi_{M}$.

Similarly, if $M$ remains enabled for another $\eta_{\max }+\delta$ time, then each professor must perform another random draw and access some counter within this interval. So there must exist another time instance $t_{2}$ such that $t_{1}<t_{2} \leq t_{1}+\eta_{\max }+\delta$, and $D_{t_{2}, p r o f . M}$ contains $a$ completely new set of random draws of size $\{$ prof. $M$ \}. Again, the probability that $M$ remains enabled after these random draws is no greater than $1-\psi_{M}$, given that the random draws in $D_{t_{1}, \text { prof.M }}$ do not cause any member of $M$ to attend a meeting. In general, the probability that $M$ remains enabled after $i$ sets of such random draws $D_{t_{1} \text { prof. } M}, D_{t_{3} \text { prof.M } M}, \ldots, D_{t_{i} \text { prof.M } M}$ is no greater than $\left(1-\psi_{M}\right)^{i}$. As $i$ tends to infinity, $\left(1-\psi_{M}\right)^{i}$ tends to zero. So the probability is zero that $M$ remains enabled forever.

Intuitively, Al requires that the $\delta$ parameter used in the algorithm is liarge enough so that a professor will not re-access a counter before the other professors get a chance to access the counter. If this assumption is removed from Theorem 3 , then a set of professors could access and re-access a counter forever without ever establishing a committee meeting. To illustrate, consider Figure 6. Each professor re-accesses a counter before the other professor could access the same counter. So no matter what committees they choose in their random draws, there just does not exist any time instance at which a professor can see the result of a counter set by the other profesisor.

The strong interaction fairness property of the algorithm additionally requires the assumption that $a$ professor's transition from thinking to waiting (see Figure 1) does not depend on the random draws performed by other processes. We refer to this assumption as A2.

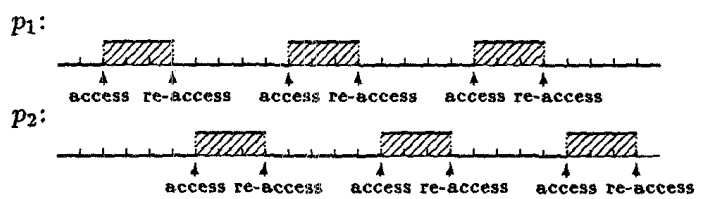

Figure 6: Two professors wait forever without establishing any meeting due to a bad choice of $\delta$.

We also need the following lemma on the probabilistic behavior of a large number of random draws.

Lemma 5 If there are infinitely many $t$ 's such that $\left\{D_{t, \text { prof. }} M\right\}=\mid$ prof. $M \mid$, then the probability is 1 that all the random draws in $D_{t, p r o f . M}$ produce the same outcome for infinitely many $t$ 's.

Proof: Let $t_{1}, t_{2}, \ldots$ be an infinite sequence of increasing time instances at each of which $\left|D_{t_{i}, \text { prof.M }}\right|=$ |prof.M|. W.l.o.g. assume that $\forall i, t_{i+1}-t_{i}>\delta$. By Lemma 2 , the sets $D_{t_{i}, \text { prof.M }}$ are pairwise disjoint.

Consider the randorn draws in set $D_{t_{i}, \text { prof.M. }}$. Let $E_{M}$ denote the event that the random draws in $D_{t, p r o f . M}$ produce the same outcome $M$. Clearly the probability of $E_{M}$ 's occurrence is independent of the time these random draws are made and is given by $\psi_{M}$. Define random variable $A_{i}$ to be 1 if $E_{M}$ occurs at $t_{i}$, and 0 otherwise. Then, $A_{i}=1$ has probability $\psi_{M}$, and $A_{i}=0$ has probability $1-\psi_{M}$.

By the Law of Large Numbers (see, for example, [6]), for any $\epsilon$ we have

$$
\lim _{n \rightarrow \infty} \mathrm{P}\left(\left|\frac{\sum_{1 \leq i \leq n} A_{i}}{n}-\psi_{M}\right| \leq \epsilon\right)=1
$$

That is, when $n$ tends to infinity, the probability is 1 that $\sum_{1 \leq i \leq n} A_{i}$ tends to $\psi_{M}$. Therefore, with probability 1 , the set $\left\{i \mid A_{i}=1, i \geq 1\right\}$ is infinite. Hence, with probability 1 , there are infinitely many $i$ 's such that the random draws in $D_{t_{i}, \text { prof.M }}$ produce the same outcome $M$.

Theorem 4 (Strong Interaction Fairness)

Assume $A 1$ and $A 2$. Then, if a committee is enabled infinitely often, with probability 1 the committee will be convened infinitely often.

Proof: Since the algorithm satisfies weak interaction fairness, we assume that there are infinitely many $i$ 's such that $M$ becomes enabled at time instance $t_{i}$. By Lemma 3 and A1, either (1) there are infinitely many $i$ 's such that each interval $\left(t_{i}, t_{i}+\theta\right]$, $\theta \geq \eta_{\max } \times \mid$ prof.M $\mid$, contains some time instance $t$ 
such that $\left|D_{t, \text { prof. } M}\right|=\mid$ prof. $M \mid$, or (2) there are infinitely many $i$ 's such that when $M$ becomes enabled at $t_{i}$, some professor $q \in$ prof. $M$ attends some meeting in the interval $\left(t_{i}, t_{i}+\theta\right)$.

Consider Case (1). By Lemma 5, with probability 1 there are infinitely many $t$ 's such that all the random draws in $D_{t, \text { prof. } M}$ produce the same outcome. So by Lemma 4 , with probability $1 M$ is convened infinitely often.

Consider Case (2). Let $D_{t_{i, q}}^{\prime}$ be the last random draw performed by $q$ before it attends some meeting in $\left(t_{i}, t_{i}+\theta\right)$. By $\mathrm{A} 2$, the random draw $D_{t_{i}, q}^{\prime}$ is independent of the enabledness of $M$ at time $t_{i}$, and so is independent of the other random draws $D_{t_{k}, q}^{\prime}$. Since the event that $D_{t_{i}, q}^{\prime}$ yields outcome $M$ has a nonzero probability, by the Law of Large Numbers (see Lemma 5), the probability is 1 that there are infinitely many $i$ 's such that $D_{t_{i}, q}^{\prime}$ yields outcome $M$. Clearly, the meeting that $q$ will attend in $\left(t_{i}, t_{i}+\theta\right)$ is determined by $D_{t_{i}, q}^{\prime}$. So, with probability $1, M$ is convened infinitely often.

Note that if Assumption A2 is dropped from Theorem 4 , then a conspiracy against strong interaction fairness can be devised. To illustrate, consider a system of two professors $p_{1}$ and $p_{2}$, and three committees $M_{1}$, which involves only $p_{1}, M_{2}$, which involves only $p_{2}$, and $M_{12}$, which involves both $p_{1}$ and $p_{2}$. Suppose that $p_{1}$ becomes waiting, and then tosses a coin to choose either $M_{1}$ or $M_{12}$. The malicious $p_{2}$ could stay in thinking until $p_{1}$ has selected $M_{1}$; then $p_{2}$ becomes waiting just before $p_{1}$ accesses $C_{M_{1}}$. Since $p_{1}$ can successfully start $M_{1}$ once it selects $M_{1}, M_{12}$ will not be started if $p_{1}$ remains in its meeting while $p_{2}$ is waiting. However, $M_{12}$ is enabled as soon as $p_{2}$ becomes waiting. So if this scenario is repeated ad infinitum, then the resulting computation would not be strong interaction fair.

The time complexity of the algorithm is analyzed in the following theorem.

Theorem 5 (Time Complexity) Suppose that from some time onward committee $M$ is enabled. Then, the expected time it takes for any member of $M$ to start a meeting is no greater than

$$
\frac{\delta+\eta_{\max }}{\psi_{M}}+\eta_{\max } \times(\mid \text { prof. } M \mid-1) .
$$

Proof: Suppose that $M$ is enabled from time $t$ onward. By Lemma 3, there exists a time instance $t_{1}, t<t_{1} \leq$ $t+\eta_{\max } \times \mid$ prof. $M \mid$, such that $\left|D_{t_{1}, \text { prof. } M}\right|=\mid$ prof. $M \mid$. By Lemma 4, if these random draws yield the same outcome $M$ (an event that occurs with probability $\psi_{M}$ ), then all professors in prof. $M$ will start a meeting of $M$ by time $t_{1}+\delta$. Otherwise, if some professor's random draw leads to the establishment of some other committee meeting involving the professor, then $M$ will also be disabled by $t_{1}+\delta$. If neither of these is the case, then each professor in prof. $M$ must perform another random draw and access the selected counter within $\eta_{\max }$ time (from the time it re-accesses the previous selected counter). So there must exist another time instance $t_{2}$, $t_{1}<t_{2} \leq t_{1}+\delta+\eta_{\max }$, such that $D_{t_{2}, \text { prof. } M}$ contains a completely new set of random draws, one by each professor in prof.M. Once again, if the new random draws yield the same outcome $M$ or cause some other committee meeting to be started, then some professor will enter a meeting by time $t_{2}+\delta \leq t_{1}+2 \delta+\eta_{\max }$. Otherwise, each professor in prof. $\bar{M}$ will perform another random draw within $\eta_{\max }$ time, and so on.

Therefore, the expected time starting from $t_{1}$ until some member of $M$ enters state $E$ to start a meeting is no greater than

$$
\begin{aligned}
& \sum_{i}\left(i \times\left(\delta+\eta_{\max }\right) \times\left(1-\psi_{M}\right)^{i-1} \times \psi_{M}\right)-\eta_{\max } \\
& =\frac{\delta+\eta_{\max }}{\psi_{M}}-\eta_{\max }
\end{aligned}
$$

Since $t_{1} \leq t+\eta_{\max } \times \mid$ prof. $M \mid$, the expected time for any member of $M$ to start a meeting when $M$ is enabled is no greater than

$$
\begin{aligned}
& \frac{\delta+\eta_{\max }}{\psi_{M}}-\eta_{\max }+\eta_{\max } \times \mid \text { prof. } M \mid \\
& =\frac{\delta+\eta_{\max }}{\psi_{M}}+\eta_{\max } \times(\mid \text { prof. } M \mid-1)
\end{aligned}
$$

Note that $\eta_{\max }$ is a constant determined by the size (number of members) of the largest committee; call this value MaxCommSize. $\psi_{M}$ is a constant determined by the maximum number of committees of which a professor can be a member; call this value MaxNumComm. Finally, $\delta$ is a constant determined by $\eta_{\max }$ and MaxCommSize. Therefore, the time complexity of the algorithm is a constant determined by MaxCommSize and MaxNumComm, and is independent of the total number of professors and committees in the system.

\section{Conclusions}

We have presented Multi, a new randomized algorithm for scheduling multiparty interactions. We have shown that by properly setting the value of $\delta$ (the amount of time a process is willing to wait for an interaction to be established), our algorithm is both weak 
and strong interaction fair with probability 1 . Our results hold even if the time it takes to access a shared variable (the communication delay) is nonnegligible. To our knowledge, this makes Multi the first algorithm for strong interaction fairness to appear in the literature.

Strong interaction fairness has been proven impossible by any deterministic algorithm. Our results therefore indicate that randomization is a feasible and efficient countermeasure to such impossibility phenomena. Furthermore, since most known fairness notions are weaker than strong interaction fairness, they too can be implemented via randomization. For example, strong process fairness [1], where a process infinitely often ready for an enabled interaction will participate in an interaction infinitely often, is also realized by our algorithm.

Multi is an extension of Francez and Rodeh's randomized algorithm for CSP-like biparty interactions. Francez and Rodeh were able to claim only weak interaction fairness for their algorithm, and then only under the limiting assumption that the communication time is negligible compared to $\delta$. In this case, strong interaction fairness wculd be possible even in a deterministic setting.

We have also analyzed the time complexity of our algorithm. Like Reif and Spirakis's real-time algorithm [1.5], the expected time taken by Multi to establish an interaction is a constant not depending on the total number of processes in the system.

Although Multi is presented in a shared-memory model, it can be easily converted to a messagepassing algorithm by letting some processes maintain the shared variables, and other processes communicate with them by message passing to obtain the values of these variables. The time to read/write a shared variable then accounts for the time it takes to deliver a message. The 6 parameter in Assumption A1 can be properly adjusted to reflect the new communication delay so that both weak and strong interaction fairness notions can still be guaranteed with probability 1 .

\section{References}

[1] K. Apt, N. Francez, and S. Katz. Appraising fairness in language:s for distributed programming. Distributed Computing, 2(4):226-241, 1988.

[2] P. Attie, I. Forman, and E. Levy. On fairness as an abstraction for the design of distributed systems. In Proc. of the 10th Int'l Conf. on Distributed Computing Systems, pages 150-157, 1990.

[3] P. Attie, N. Francez, and O. Grumberg. Fairness and hyperfairne:ss in multi-party interactions. Distributed Computing, 6:245-254, 1993.
[4] R. Bagrodia. Process synchronization: Design and performance evaluation of distributed algorithms. IEEE Trans. on Software Engineering, SE-15(9):10531065 , Sept. 1989.

[5] K. Chandy and J. Misra. A Foundation of Parallel Program Design. Addison-Wesley, 1988.

[6] K. Chung. A Course in Probability Theory. A Series of Monographs and Textbooks. Academic Press, New York, second edition, 1974.

[7] N. Francez and M. Rodeh. A distributed abstract data type implementied by a probabilistic communication scheme. Technical Report TR-80, IBM Israel Scientific Center, Apr. 1980. Also: Proc. of the 21st Annual IEEE Symp. on Foundations of Computer Science, pages 373-379, 1980.

[8] Y.-J. Joung. Characterizing fairness implementability for multiparty interaction. In Proc. of the 23rd Int'l Colloquium on Automata, Languages and Programming, 1996 (to appear).

[9] Y.-J. Joung and S.A. Smolka. Coordinating first-order multiparty interactions. ACM Trans. on Programming Languages and Systems, 16(3), May 1994.

[10] Y.-J. Joung and S.A. Smolka. A comprehensive study of the complexity of multiparty interaction. Journal of the ACM. To appear.

[11] D. Kumar. An implementation of N-party synchronization using tokens. In Proc. of the 10th Int'l Conf. on Distributed Computing Systems, pages 320$327,1990$.

[12] D. Lehman and M. Rabin. On the advantage of free choice: A symmetric and fully distributed solution to the dining philosophers problem (extended abstract). In Proc. of the 8th ACM Symp. on Principles of Programming Languages, pages 133-138, 1981.

[13] M. Park and M. Kim. A distributed synchronization scheme for fair multi-process handshakes. Information Processing Letters, 34:131-138, Apr. 1990.

[14] S. Ramesh. A new and efficient implementation of multiprocess synchronization. In Proc. Conf. on PARLE, Lecture Notes in Computer Science 259, pages 387-401, 1987.

[15] J. Reif and P. Spirakis. Real time synchronization of interprocess cornmunications. ACM Trans. on Programming Languages and Systems, 6(2):215-238, Apr. 1984.

[16] P. Sistla. Distributed algorithms for ensuring fair interprocess communications. In Proc. of the $3 r d A C M$ Symp. on Principles of Distributed Computing, pages 266-277, 1984.

[17] Y.-K. Tsay and R. Bagrodia. A real-time algorithm for fair interprocess synchronization. In Proc. of the 12th Int'l Conf. on Distributed Computing Systems, pages 716-723, 1992.

[18] Y.-K. Tsay and R. Bagrodia. Some impossibility results in interprocess synchronization. Distributed Computing, 6(4):221-231, 1993. 